Mario Krali ${ }^{*}$, Andrey Gurov ${ }^{* *}$

\section{Analysis of Granularity Adjustment for Regulatory Capital}

\begin{abstract}
The lack of portfolio granularity in terms of exposure has been shown to have important implications for the amount of a financial institution's economic capital. Based on a numerical simulation model, we provide concrete examples of how granularity affects capital levels. We achieve this by following two simulation approaches, including a dynamic setup as a more realistic version of the analysis. We show that granularity has an indirect effect on the expected loss component. This could lead to significant changes in the competitive environment should banks consider adding a granularity adjustment to the estimated amount of capital and account for it in their pricing.
\end{abstract}

Keywords: Finance, Regulation, Basel, Granularity, Banks, Capital, Concentration, Risk, Corporate Exposures.

JEL codes: E58, G21, G28, G32.

\author{
${ }^{*}$ Technische Universität \\ München, Munich, \\ Germany \\ E-mail: \\ mario.krali@tum.de \\ ** American University in \\ Bulgaria, Blagoevgrad, \\ Bulgaria \\ E-mail (corresponding author): \\ agurov@aubg.edu
}

\title{
Introduction
}

The topic of this paper revolves around capital requirements of Financial Institutions (FIs). Throughout the paper we make use of the granularity adjustment introduced by Gordy (2003) and Gordy and Lütkebohmert (2013). Its purpose was to augment the output of the asymptotic single risk factor (ASRF) model by 
adjusting for portfolio granularity when computing the level of capital a FI would need. We apply the granularity adjustment model in order to analyze the deviations from the asymptotic model across different portfolio sizes and structures and judge the magnitude of necessary adjustments to the calculated capital levels under the Basel framework. We further examine the effects that such capital adjustments could have on the FI's stability and pricing policy.

\section{The Role of Capital Requirement}

The amount of capital that an institution holds is a key factor in credit risk management. Its main function is to serve as a cushion against unexpected adverse scenarios and thus prevent an FI from going bankrupt. On the other hand, FIs treat expected losses (EL) as operating costs by estimating the average amount losses over an extended period of time and accounting for it in their loan prices. Should an institution experience higher than the expected amount of loss in any given year, it would need to be absorbed by a sufficient amount of own capital or otherwise the FI will be forced into economic insolvency, i.e. higher liabilities than available assets.

In order to estimate the unexpected loss, a standard best-practice approach is to make use of the value-at-risk measure ( $\mathrm{VaR})$. VaR is a measure of the maximum variation of the losses from their expected value at a certain confidence level. In essence, by calculating the VaR FIs estimate the possible deviation of losses from the expected level in an adverse scenario (e.g. one that occurs on average once in a thousand years). The close relation between VaR and capital need stands in the fact that based on the value at risk an institution can decide on the amount of capital it would need in order to survive a particular bad state of the world. A bank can thus be considered as safer in the sense that it has the ability to absorb losses higher than the expected amount. Given its important risk-absorbing properties, the amount of capital has been the main focus of discussion in the Basel Committee of Banking Supervision.

\section{VaR Models: CreditRisk+ Vs. CreditMetrics}

Many credit risk models make use of the VaR framework. Two of the most wellknown are CreditRisk+ by Credit Suisse and J.P.Morgan's CreditMetrics. While both use VaR in estimating capital requirements, the two models differ in the particular approaches they follow. In the case of CreditRisk+, the model takes into consideration only two states of the world: the good state (survival of the obligor) and the bad one (default). It does not make any assumptions about the 
causes of default (Credit Suisse First Boston, 1997). On the other hand, CreditMetrics does not only allow for these two states, but also takes into account scenarios between the two extremes in the form of credit rating changes. In addition, CreditMetrics is based on the assumption that the systematic risk factor is normally distributed (Gupton, Finger and Bhatia, 1997). Gordy (2000) points out that differences in the distribution assumptions lead eventually to inconsistencies in the probabilities of default. However, the author argues that there exists a mapping between the models, such that under appropriate assumptions both models arrive at the same conclusions.

\section{Initiation of the Basel Protocol}

The first international attempt towards achieving a higher level of preparedness of the global financial system against severe adverse developments was the initiation of the Basel Protocol, starting with Basel I in 1988. Since then, the implementation of the Basel Protocol has had a sweeping impact across the global financial system. Its purpose was to make sure that the financial system was properly capitalized in order to survive a major shock. In order to achieve that, Basel I imposed a minimum requirement of $8 \%$ capital to risk-weighted-assets ratio (Basel Committee on Banking Supervision, 2014). It soon became clear, however, that the first protocol must eventually be redesigned, so that it allows for a more risk-sensitive approach to the calculation of minimum capital requirements.

The second Basel Protocol, namely Basel II, provided the system with new criteria, but it also introduced new approaches for the purpose of risk estimation. Its most important innovation was in the implementation of the three-pillar approach to banking regulation (Basel Committee on Banking Supervision, 2014). Pillar one introduced the internal ratings-based (IRB) approach, besides the existing standardized approach for calculating the minimum capital requirements. Pillar two consisted of the continuous supervision of the FIs by the regulators in order to ensure their capital adequacy. The third Pillar emphasized the importance of market discipline and consisted of requirements regarding the FI's disclosure of information.

The most important innovation of Basel II by far was the introduction of the IRB approach. By giving FIs the option to use their own estimates of risk measure parameters ( $P D$-probability of default; $L G D$-loss given default; $E A D$-exposure given default, etc.), the Basel committee intended for banks to utilize their internal expertise and data in order to come up with a better approximation for their portfolios' riskiness. 


\section{Risk Sources}

When analysing loan portfolios it is important to distinguish between two sources of risk: the systemic d-dimensional risk factor $X=\left(x_{1}, x_{2}, \ldots, x_{d}\right)$, and the idiosyncratic one (dependent on the obligor). The theoretical and mathematical basis of Basel II originated from a model developed by Oldrich Vasicek. In his model, known as the Vasicek Single Risk Factor model, the systematic risk factors are grouped into a one-dimensional vector, thus $X=x$. In addition, the systematic risk factor is assumed to be normally distributed. Originally developed for modeling the behaviour of interest rates, Vasicek extended the single factor model in order to derive a loss rate distribution function across portfolios of loans (Vasicek, 1991).

The slightly modified version of the Vasicek model, upon which Basel II was built, was developed by Gordy (2003). In order to achieve portfolio-invariant capital requirements, Gordy makes the important assumption that the loan portfolio is fine-grained. Denoting the set of exposures by $A=\left\{A_{i}: i \in N\right\}$ where $A_{i}$ stands for $E A D$ to a given obligor, $\mathrm{s}_{\mathrm{i}}$ for the share of the latter, and $\mathrm{n}$ for the number of customers, this implies:

$s_{i}=\frac{A_{i}}{\sum_{i=1}^{n} A_{i}} \underset{n \rightarrow \infty}{\rightarrow} 0$

Hence, no exposure is assumed to have a significant size relative to the rest of the portfolio, which effectively eliminates the idiosyncratic (concentration) risk factor and makes the loss function portfolio-invariant. The drawback of this assumption, on the other hand, is the tendency of the model to underestimate the regulatory (or economic) capital, which is clearly dependent on concentration risk.

\section{Concentration Risk}

According to the Basel Committee on Banking Supervision (Basel Committee on Banking Supervision, 2006) concentration risk originates from one of the following:

- small size of the portfolio,

- relatively large exposure with respect to a single obligor,

- contagion effect (inter-related firms). 
In this paper we examine the concentration risk resulting from the first two factors, which is not explicitly dealt with in the ASRF model. We later analyse how this 'abuse' occurs and the effects it has on capital requirements. In the Bank for International Settlements' (BIS) document, the Basel Committee, by undertaking surveys, recognizes the regulatory challenge in terms of not only measuring, but also accounting for concentration risk (Basel Committee on Banking Supervision, 2006).

One way to account for concentration risk originating from large exposures is with the use of a granularity adjustment $(G A)$. The $G A$ is a measure of the deviation between the capital requirement calculated as in Basel under the assumptions of the ASRF and the true one accounting for concentration risk. Intuitively, the adjustment accounts for the error a FI would make by assuming a fine-grained portfolio. Once the $G A$ is specified, the FI can analyze the difference from the ASRF capital amount and hence, create a concentration risk measure based on capital as an indicator.

Basel III recently introduced the Liquidity Coverage Ratio (LCR) in order to promote short term readiness on the side of the FI in term of its liquidity risk profile (Basel Committee on Banking Supervision, 2013). By aiming at reducing contagion (spillover) effect, the LCR is hence related to concentration risk via the third factor listed above. However, in a recent study on LCR, Brůna and Blahová (2016) show that while the LCR leads to significant changes in a FI's liquidity allocation, it may also underestimate its liquidity positions and thus lead to liquidity allocation inefficiencies.

\section{Effects of Accounting for Granularity}

The inclusion of more risk measures is shown to have a significant effect on the profitability of a FI (Stein, 2005). By deriving the cost functions for models of different predictive power (in terms of PDs) Stein concludes that the inclusion of more risk measures in the predictive model results in lower default costs relative to the weaker models. By scanning the risk of the borrower in a more accurate way, the FI gains competitiveness in risk pricing, thus being able to cherry-pick its portfolio at the expense of less advanced institutions. Subsequently, it is important to examine whether the inclusion of concentration risk in risk pricing has a comparable effect in cost reduction, revenue generation, and profitability of a FI. 


\section{The Model}

\section{Granularity Adjustment}

Preserving its main assumptions, and building upon Vasicek's SFM, Gordy (2003) extends the model into the Asymptotic Single Risk Factor (ASRF) model. The author considers the loss function due to defaulting obligors of a FI and proves, under the Law of Large Numbers (LLN), that the percentile of the risk factor matches that of the amount of loss. Denoting $U_{i}$ for loss rate (a stochastic term), and $s_{i}$ for the individual exposures one finds $L_{n}$ :

$$
L_{n}=\sum_{i=1}^{n} s_{i} U_{i}
$$

Additionally, Gordy assumes that $\mu(x)=E\left[L_{n} \mid X=x\right]$ is an increasing function of $X$. Letting $\alpha_{q}(Y)$ stand for quantile function of a random variable we get that:

$$
\left|\alpha_{q}\left(L_{n}\right)-E\left[L_{n} \mid X=\alpha_{q}(X)\right]\right| \underset{n \rightarrow \infty}{\rightarrow} 0
$$

Intuitively this means that $\alpha_{q}\left(L_{n}\right)$ converges to $\mu\left(\alpha_{q}(X)\right)$. The difference $\mid \alpha_{q}\left(L_{n}\right)$ $\mu\left(\alpha_{q}(X)\right) \mid$ is the granularity adjustment attributed to the "chunky" portfolio. For a rigorous treatment of the above see Gordy (2003).

Using this result, the capital requirement for an individual loan is measured in terms of the probability of the risk factor's level. Hence, the ASRF model represents an important and simplistic credit risk model that performs well under the assumption that the exposures of a portfolio are evenly spread amongst the individual obligors (Gordy, 2003). As long as there is a big number of customers ( $n$ sufficiently large) the size of the individual exposures $A_{i}$ will not matter and the probability of default $(P D)$ distribution will approach asymptotically that of the ASRF (see equation (1)).

On the other hand, this intuition only performs well (limit as $n \rightarrow \infty$ when considering portfolios with many exposures i.e. $n=10,000$. In cases where $\mathrm{n}$ is a smaller number, one cannot assume a fine-grained portfolio, so the relative weights of some exposures are different from zero, thus creating exposure to concentration risk.

This major shortcoming of the ASRF model could potentially give rise to significant idiosyncratic (obligor-specific) risk in certain FIs. In such instances, the 
assumption that the real loss function converges in distribution to the asymptotic case does not hold, which compromises the capital adequacy (dependent on the percentile of the risk factor) of the institutions and threatens the stability of the broader financial system.

The difference between the actual $\mathrm{VaR}$ and the asymptotic one can be approximated by a Taylor series expansion centred around the asymptotic portfolio loss (Gordy and Lütkebohmert, 2013). Given the parameters that are needed in order to estimate the moments of loss given default ( $L G D)$, GA becomes a function dependent on the number of exposures. The following simplified version of GA shows the factors that influence the adjustment:

$$
\begin{aligned}
G A & =\frac{1}{2 \mathcal{K}^{*}} \sum_{i=1}^{n} s_{i}^{2}\left[\left(\delta \mathcal{C}_{i}\left(\mathcal{K}_{i}+\mathcal{R}_{i}\right)+\delta\left(\mathcal{K}_{i}+\mathcal{R}_{i}\right)^{2} \frac{V\left[L G D_{i}\right]}{E\left[L G D_{i}\right]^{2}}\right)\right. \\
& \left.-\mathcal{K}_{i}\left(\mathcal{C}_{i}+2\left(\mathcal{K}_{i}+\mathcal{R}_{i}\right) \frac{V\left[L G D_{i}\right]}{E\left[L G D_{i}\right]^{2}}\right)\right]
\end{aligned}
$$

Here, the terms $s_{i}, K_{i}$ stand for share of exposure and capital requirement for the $i^{\text {th }}$ obligor, respectively and $\mathcal{K}^{*}=\sum_{\mathrm{i}=1}^{\mathrm{n}} \mathrm{s}_{\mathrm{i}} \mathcal{K}_{\mathrm{i}}$. As GA depends on $s_{i}$ we see that the portfolio invariance assumption does not hold in this setup. The parameters $\gamma, \delta$ are regulatory parameters which we set to $\gamma=0.25$ and $\delta=4.83$ following the Basel CP2 (Gordy and Lütkebohmert, 2013). The precision parameter is set to $\xi=0.25$ an $E[L G D]$, which stands for expected loss given default and is set to its regulatory value of 0.45 .

Table 1: Components of the Granularity Adjustment Equation (2)

$$
\begin{aligned}
& \mathcal{R}_{i}=E\left[L G D_{i}\right] P D_{i} \\
& \mathcal{C}_{i}=\frac{V\left[L G D_{i}\right]+E\left[L G D_{i}\right]^{2}}{E[L G D]} \\
& V\left[L G D_{i}\right]=\gamma E\left[L G D_{i}\right]\left(1-E\left[L G D_{i}\right]\right) \\
& \mathcal{K}_{i}=E\left[U_{i} \mid X=\alpha_{q}(X)\right]-E\left[U_{i}\right]=E\left[L G D_{i}\right] P D_{i} \omega_{-} i\left(\alpha_{q}(X)-1\right)
\end{aligned}
$$

Considering cases when the data might be incomplete, Gordy and Lütkebohmert (2013) derive maximum and minimum bounds for the granularity adjustment. This can be achieved both for a heterogeneous or homogenous portfolio structure. The mapping of a heterogeneous portfolio into a homogeneous one 
is achieved by making transformations in four parameters, namely the share of exposure, the risk factor loading, the number of exposures in the transformed new homogenous portfolio, and the new variance of $L G D$. The importance of the upper and lower bounds stands in the fact that calculating the exact $G A$ is difficult to implement in real life given the amount and variation in the parameters it uses as an input.

\section{Simulation Setup}

We use a methodology, which is based on hypothetical portfolios of corporate exposures that typically display a large variation in terms of size. In order to achieve representativeness and comparability of the results, we partly calibrate the portfolio characteristics to those of Gordy and Lütkebohmert (2013). As for the distribution of the borrowers across rating grades, we assume a structure that follows a log-normal distribution, which is observed in real-life banks' portfolios (Unicredit Bulbank, 2012). Based on the generated portfolios, we simulate the respective loss distribution function.

\section{Portfolio Structure}

Since one of our objectives is to demonstrate the negative relation between GA and the number of exposures, we consider portfolios of four different sizes, namely $1000,2000,3000$, and 6000 exposures. Each of the portfolios is constructed based on the same distributions in terms of the pair $\left(P D_{i}, E A D_{i}\right)$.

\section{Rating Grades}

We set the rating grades to follow a log-normal distribution. Each of the portfolios is assumed to have 17 rating classes starting from AAA to C. The portfolios represent only the performing loans of an institution and thus do not include already defaulted borrowers. The PD bands are set in accordance to the table (2) below (Featherstone, Roessler, and Barry, 2006): 
Table 2: Rating Grades' Probabilities

\begin{tabular}{ccc}
\hline Rating Grade & Lower Bound & Upper bound \\
\hline AAA & $0.000 \%$ & $0.020 \%$ \\
\hline AAt & $0.020 \%$ & $0.030 \%$ \\
\hline AA & $0.030 \%$ & $0.040 \%$ \\
\hline AA- & $0.040 \%$ & $0.050 \%$ \\
\hline A+ & $0.050 \%$ & $0.070 \%$ \\
\hline A & $0.070 \%$ & $0.090 \%$ \\
\hline A- & $0.090 \%$ & $0.140 \%$ \\
\hline BBB & $0.140 \%$ & $0.210 \%$ \\
\hline BBB & $0.210 \%$ & $0.310 \%$ \\
\hline BBB- & $0.310 \%$ & $0.520 \%$ \\
\hline BB+ & $0.520 \%$ & $0.860 \%$ \\
\hline BB & $0.860 \%$ & $1.430 \%$ \\
\hline BB- & $1.430 \%$ & $2.500 \%$ \\
\hline B & $2.500 \%$ & $4.305 \%$ \\
\hline B & $4.305 \%$ & $8.611 \%$ \\
\hline
\end{tabular}

In order to ensure that the $P D$ s follow a log-normal distribution, we first generate random variables which are normally distributed $Y \sim N(\mu, \sigma)$ and then take their exponential $\left(e^{Y}\right)$, so that for the individual PDs we get: $P D_{i}=e^{Y i}$. As a mean and standard deviation of the log-normal distribution we set the following values: $\mu^{\prime} \approx 1.17 \%$ and $\sigma^{\prime} \approx 2.31 \%$. These parameters are then converted based on the following formulas:

$\mu=\ln \left(\frac{\mu^{\prime 2}}{\sqrt{\left(\sigma^{\prime}+\mu^{2}\right)}}\right)$ and $\sigma=\sqrt{\ln \left(\frac{\sigma^{\prime}}{\mu^{\prime 2}}+1\right)}$

Using the above parameters we first simulate the unconditional probabilities of default $P D_{i}$ for each obligor $i=1, \ldots, n$. Below we illustrate a typical distribution across the different $P D$-rating classes. 


\section{Figure 1: Distribution of Rating Grades}

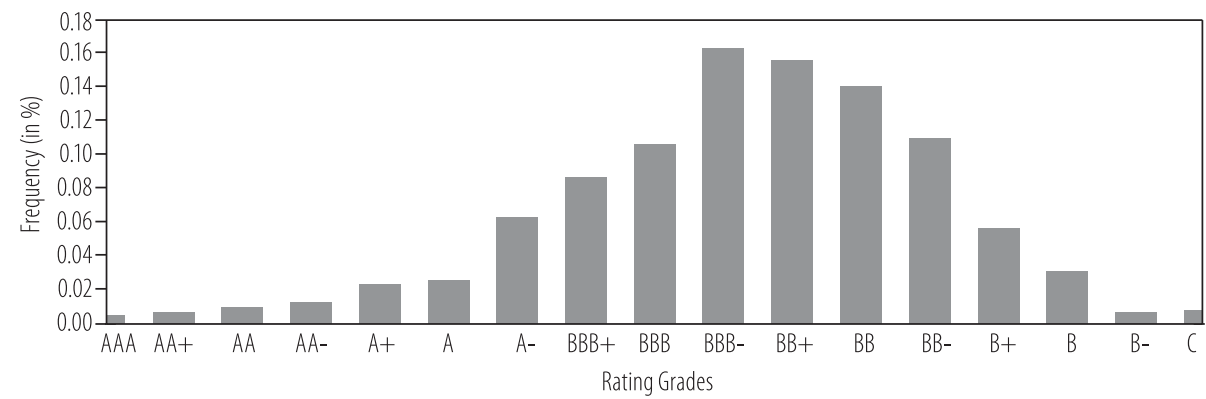

As seen above, the procedure results in a relatively safe portfolio with less than $5 \%$ of the borrowers in the grades $\mathrm{B}, \mathrm{B}-$, and $\mathrm{C}$, which is representative of a typical mid-size institution.

\section{Exposures}

Once we have assigned $P D s$, we proceed to generate the exposures. In order to set up a realistic portfolio, we take into account the dependence between exposure amount and $P D s$ and set the distribution within each $P D$-band as log-normal. Thus, for the exposures we get to be the following:

$A_{i}=e^{Z_{i}}$ where $Z \sim \mathcal{N}\left(\mu_{A}, \sigma_{A}\right)$ and $\mu_{A}{ }^{\prime}=E\left[A \mid P D_{i}\right]$ and $\sigma_{A}{ }^{\prime}=\sigma_{A}{ }^{\prime}\left(P D_{i}\right)$

We assume that, following standard risk-management practices, both the mean and the variance of the exposures are negatively related to the $P D s$, thus:

$$
\frac{\partial \mu_{A}{ }^{\prime}}{\partial P D}<0 \text { and } \frac{\partial \sigma_{A}{ }^{\prime}}{\partial P D}<0
$$

Regarding the calibration in terms of exposures, we build our simulation according to the structure in Table 3. In order to proceed we need to convert each of the parameters in the table accordingly by making use of the two formulas in equations (3). 
Table 3: Portfolio Structure (mean, standard deviation) in euros

\begin{tabular}{|c|c|c|c|c|}
\hline & \multicolumn{2}{|c|}{$P(2000,3000,6000)$} & \multicolumn{2}{|c|}{$P(1000)$} \\
\hline & Mean & St.Dev & Mean & St.dev \\
\hline AAA & $€ 15,000,000.00$ & $€ 25,000,000.00$ & $€ 15,000,000.00$ & $€ 25,000,000.00$ \\
\hline $\mathrm{AA}+$ & $€ 15,000,000.00$ & $€ 25,000,000.00$ & $€ 15,000,000.00$ & $€ 25,000,000.00$ \\
\hline AA & $€ 15,000,000.00$ & $€ 25,000,000.00$ & $€ 15,000,000.00$ & $€ 20,000,000.00$ \\
\hline AA- & $€ 12,000,000.00$ & $€ 20,000,000.00$ & $€ 12,000,000.00$ & $€ 20,000,000.00$ \\
\hline A+ & $€ 10,000,000.00$ & $€ 20,000,000.00$ & $€ 10,000,000.00$ & $€ 15,000,000.00$ \\
\hline A & $€ 10,000,000.00$ & $€ 20,000,000.00$ & $€ 10,000,000.00$ & $€ 15,000,000.00$ \\
\hline A- & $€ 8,000,000.00$ & $€ 20,000,000.00$ & $€ 8,000,000.00$ & $€ 15,000,000.00$ \\
\hline $\mathrm{BBB}+$ & $€ 8,000,000.00$ & $€ 15,000,000.00$ & $€ 6,000,000.00$ & $€ 15,000,000.00$ \\
\hline BBB & $€ 5,000,000.00$ & $€ 10,000,000.00$ & $€ 5,000,000.00$ & $€ 10,000,000.00$ \\
\hline BBB- & $€ 4,000,000.00$ & $€ 10,000,000.00$ & $€ 4,000,000.00$ & $€ 10,000,000.00$ \\
\hline $\mathrm{BB}+$ & $€ 3,000,000.00$ & $€ 5,000,000.00$ & $€ 3,000,000.00$ & $€ 5,000,000.00$ \\
\hline $\mathrm{BB}$ & $€ 3,000,000.00$ & $€ 5,000,000.00$ & $€ 3,000,000.00$ & $€ 5,000,000.00$ \\
\hline BB- & $€ 3,000,000.00$ & $€ 4,000,000.00$ & $€ 3,000,000.00$ & $€ 4,000,000.00$ \\
\hline $\mathrm{B}+$ & $€ 3,000,000.00$ & $€ 4,000,000.00$ & $€ 3,000,000.00$ & $€ 4,000,000.00$ \\
\hline B & $€ 3,000,000.00$ & $€ 4,000,000.00$ & $€ 3,000,000.00$ & $€ 4,000,000.00$ \\
\hline$B-$ & $€ 3,000,000.00$ & $€ 4,000,000.00$ & $€ 3,000,000.00$ & $€ 4,000,000.00$ \\
\hline C & $€ 3,000,000.00$ & $€ 4,000,000.00$ & $€ 3,000,000.00$ & $€ 4,000,000.00$ \\
\hline
\end{tabular}

Once we generate the exposures we observe the distribution of a typical portfolio across the rating classes (relative share per grade).

\section{Figure 2: Exposure-Weighted Distribution}

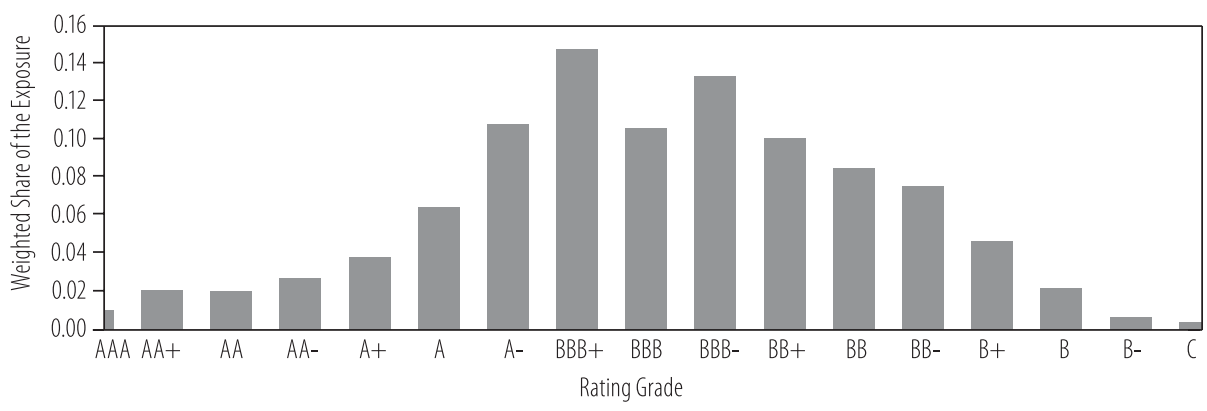

\section{Granularity Adjustment and the Monte Carlo Simulation}

In order to examine and quantify the relation between $G A$ and the portfolio size we apply two approaches. The first one is closely linked to the ASFR model and 
assumes a static portfolio with the only uncertainty stemming from the realization of the systemic factor. Even though this is not a realistic representation of what is observed in reality, we employ this approach for the sake of comparability with the ASFR model.

In the second approach we allow our portfolios to change stochastically over time. Thus while initially we have a given 'vector' of borrowers, say $P_{n}\left(t_{0}\right)=\left(p_{1}\left(t_{0}\right)\right.$, $\left.p_{2}\left(t_{0}\right), \ldots, p_{n}\left(t_{0}\right)\right)$, at the end of the simulation we get a 'quasi-permutation' of the vector entries, $P_{n}\left(t_{1}\right)=\left(p_{1}\left(t_{1}\right), p_{2}\left(t_{1}\right), \ldots, p_{n}\left(t_{1}\right)\right)$. We use the same distribution in generating the random variables, which preserves the portfolio structure, yet allows for changes in individual entries. This corresponds to the normal processes of retirement and acquisition of new customers as well as the rating migrations and exposure changes within the set of existing customers.

In other words, we have two processes with respect to time $t$, namely, $A: X_{t_{0}} \rightarrow X_{t_{1}}$ (risk factor simulation) and $B: P_{n\left(t_{0}\right)} \rightarrow P_{n\left(t_{1}\right)}$ (portfolio mapping). With this setup we proceed to tackle the question: given $t$ scenarios which simulate the condition of the economy in the following year, what is the resulting distribution of portfolio losses? In both approaches we make use of Monte-Carlo simulations in order to generate the loss distribution. By repeatedly simulating the systematic risk factor $X=x$, we replicate possible real-life scenarios, and thus the realized amounts of loss.

Specifically, we follow a similar procedure to Gordy (2003). The ASRF model assumes the risk factor to be normally distributed $N(0,1)$. So far, in our model we have the unconditional $P D s$, where $P D$ represents the expected $P D$ of borrower $\mathrm{i}$ with respect to $X$. Hence, $P D_{i}=E\left[P D_{i} \mid X\right]$. In order to account for the behavior of borrowers under an extreme scenario we consider the mapping of each of the unconditional PDs into conditional ones, i.e. given the realization of the risk factor. The mapping function is derived following the Merton (1974) model similarly to the ASRF model: the return $R_{i}$ of an asset (loan in our case) according to Merton is given by $R i=\psi i \epsilon_{i}-X \omega_{i}$, where $\omega_{i}$ is a risk factor weight. In order for the obligor to default we have $R_{i}$ falling below a threshold value $\gamma_{i}$. Arranging the equation in order to get the i.i.d $N(0,1)$ term $\epsilon_{i}$ to the one side, and then applying the probability distribution function we get:

$$
p_{i}(x)=P\left[\epsilon_{i} \leq \frac{\left(\gamma_{i}+x \omega_{i}\right)}{\psi_{i}}\right]
$$

Noting the distribution of $\epsilon_{i}$ we get: 


$$
p_{i}(x)=\Phi\left[\frac{\left(\gamma_{i}+x \omega_{i}\right)}{\psi_{i}}\right]
$$

We let $\gamma_{i}=\Phi^{-1}\left(P D_{i}\right)$ where $\Phi^{-1}$ corresponds to the inverse normal distribution and $\psi_{i}$ is given by:

$$
\psi_{i}=\sqrt{1-\omega_{i}^{2}}
$$

And for the weights we have:

$$
\omega_{i}=\sqrt{\rho_{i}}
$$

Note that $\rho_{i}$ stands for the correlation of the borrower with respect to the risk factor. The correlations are found based on the Basel II criteria as a function of the $P D$ with the use of the following formula:

$$
\rho=\left(0.12\left(1-e^{-50 \mathrm{PD}_{i}}\right) /\left(1-e^{-50}\right)\right)+0.24\left(1-\left(1-e^{-50 \mathrm{PD}_{i}}\right) /\left(1-e^{-50}\right)\right)
$$

At each trial of the simulation, we simulate the risk factor $X$. For each borrower we then draw independent random numbers (uniformly distributed in the closed set $[0,1]$ ), based on which we let each of the borrowers default with the corresponding conditional probability of default. We perform 10,000 simulations for each examined portfolio. In case of ambiguous results, we double the number of the simulations. The granularity adjustment is calculated as $\left(\alpha_{.999}(L)-K\right)$.

\section{Results}

In this section we present the results from the Monte Carlo simulation and the Granularity Adjustment for both cases of dynamic and fixed portfolios.

\section{Dynamic Portfolios}

Due to the high number of required simulations, we perform our dynamic procedure on three portfolios, consisting of 1,000, 2,000, and 3,000 customers, respectively denoted by $P(1000), P(2000)$, and $P(3000)$. In all three cases we observe that there is indeed a difference between the Basel II prescribed level of capital $K$ and the simulated amount of unexpected loss based on the 99.9 percentile. 
Table 4: Dynamic Portfolios Results (GA calculated by formula according to Gordy)

\begin{tabular}{|c|c|c|c|}
\hline & $P(1000)$ & $P(2000)$ & $P(3000)$ \\
\hline Percentiles & $G A$ & $G A$ & GA \\
\hline 99.85 & $€ 25,407,289$ & $€ 24,353,848$ & $€ 25,487,045$ \\
\hline 99.86 & $€ 20,500,716$ & $€ 20,384,191$ & $€ 21,405,581$ \\
\hline 99.87 & $€ 14,719,470$ & $€ 22,608,650$ & $€ 35,620,593$ \\
\hline 99.88 & $€ 25,162,009$ & $€ 32,552,270$ & $€ 23,837,024$ \\
\hline 99.89 & $€ 25,092,955$ & $€ 31,535,973$ & $€ 20,060,998$ \\
\hline 99.9 & $€ 15,371,922$ & $€ 20,464,457$ & $€ 30,124,074$ \\
\hline 99.91 & $€ 29,215,812$ & $€ 28,366,997$ & $€ 22,364,146$ \\
\hline 99.92 & $€ 26,106,688$ & $€ 22,975,428$ & $€ 17,243,649$ \\
\hline 99.93 & $€ 21,218,952$ & $€ 27,440,460$ & $€ 28,562,812$ \\
\hline 99.94 & $€ 25,780,281$ & $€ 24,833,826$ & $€ 26,334,157$ \\
\hline \multirow[t]{2}{*}{99.95} & $€ 18,010,898$ & $€ 30,731,524$ & $€ 30,203,776$ \\
\hline & $K-U L$ & $K-U L$ & $K-U L$ \\
\hline 99.85 & $€-20,032,544$ & $€ 23,752,96$ & $€ 100,012,913$ \\
\hline 99.86 & $€-42,273,652$ & $€ 14,743,331$ & $€ 74,851,185$ \\
\hline 99.87 & $€-71,026,496$ & $€ 44,402,164$ & $€ 61,411,769$ \\
\hline 99.88 & $€-50,629,516$ & $€ 16,952,477$ & $€ 39,431,005$ \\
\hline 99.89 & $€-55,112,546$ & $€ 35,273,773$ & $€ 17,037,945$ \\
\hline 99.9 & $€-78,012,613$ & $€-32,416,945$ & $€-9,206,630$ \\
\hline 99.91 & $€-22,777,533$ & $€-100,355,217$ & $€-20,238,957$ \\
\hline 99.92 & $€-53,449,540$ & $€-153,395,335$ & $€-26,408,657$ \\
\hline 99.93 & $€-77,091,124$ & $€-139,083,792$ & $€-61,002,011$ \\
\hline 99.94 & $€-79,307,483$ & $€-151,278,775$ & $€-93,205,441$ \\
\hline 99.95 & $€-126,283,823$ & $€-159,566,538$ & $€-108,642,968$ \\
\hline
\end{tabular}

While the number of simulations needs to be increased in order to generate monotonicity in the results, our simulations show clearly that there is an increasing discrepancy (and thus the need to adjust for the lack of granularity) as the number of exposures decreases.

Despite representing the more realistic dynamic portfolio setup, these results do not allow for a direct comparison between the simulated loss amounts and the granularity adjustment suggested by Gordy and Lütkebohmert (2013) as the latter is based on a fixed portfolio structure in the individual borrower characteristics. We consider this case in the following section. 


\section{Fixed Portfolios}

In this section, the above procedure is repeated by selecting at random a representative portfolio for each portfolio size and keeping it fixed over time $t$.

For all four portfolio sizes under consideration, namely $\mathrm{P}(1000), \mathrm{P}(2000), \mathrm{P}(3000)$, $\mathrm{P}(6000)$, we see a discrepancy in the capital requirement (Basel) and the realized amount of unexpected loss, similarly to above.

Figure 3: Loss Distribution for 1000 Borrowers

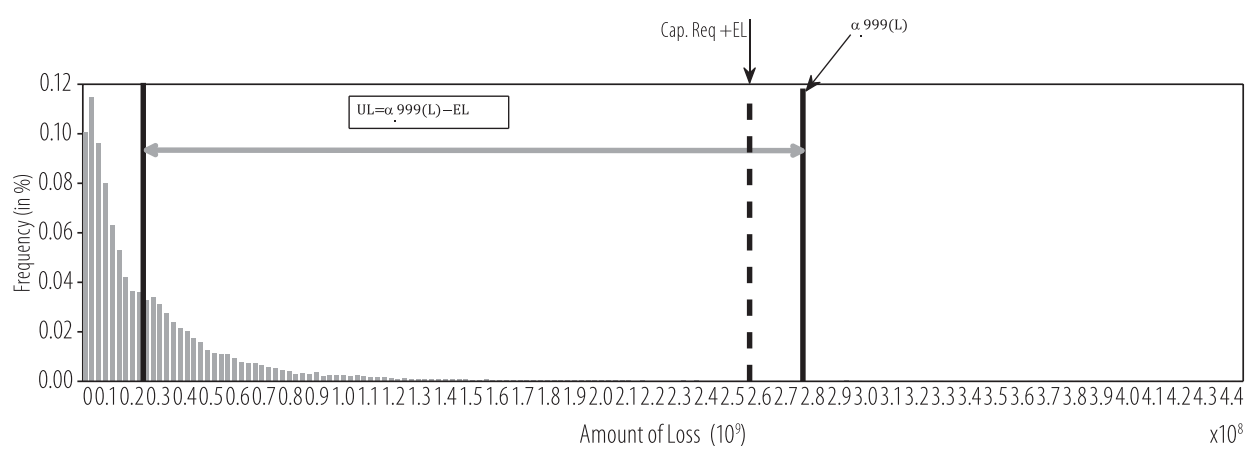

Figure 4: Loss Distribution for 2000 Borrowers

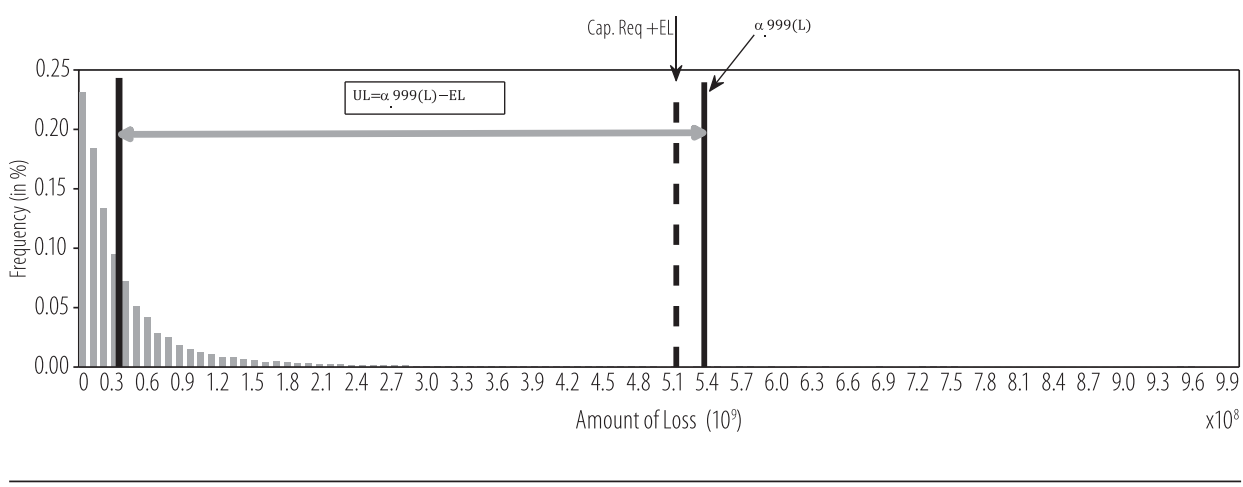


Figure 5: Loss Distribution for 3000 Borrowers

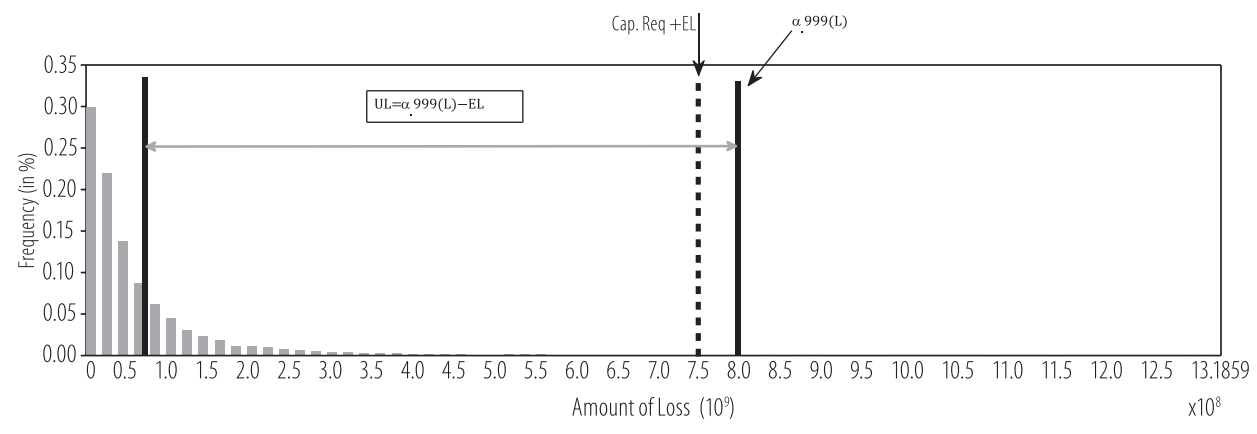

Figure 6: Loss Distribution for 6000 Borrowers

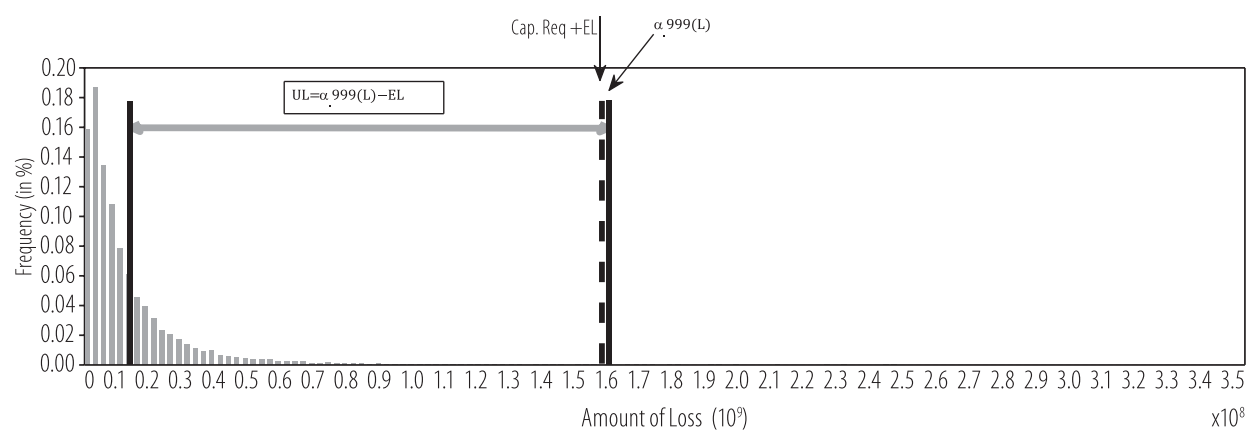

Table (5) provides GA calculated via the Gordy's formula and the amounts by which the considered portfolios are undercapitalized.

Table 5: GA and the realized granularity difference at the $99.9^{\text {th }}$ percentile

\begin{tabular}{ccccc}
\hline & $G A(P(1000))$ & $G A(P(2000))$ & $G A(P(3000))$ & $G A(P(6000))$ \\
\hline Percentile & $€ 19,133,312$ & $€ 24,719,738$ & $€ 26,092,012$ & $€ 24,394,455$ \\
\hline 0.9988 & $€-5,104,519$ & $K-U L$ & $K-U L$ & $K-U L$ \\
\hline 0.9989 & $€-12,621,738$ & $€ 9,059,568$ & $€ 28,358,276$ & $€ 129,546,200$ \\
\hline 0.9990 & $€-20,196,117$ & $€-16,066,590$ & $€-3,234,769$ & $€ 85,176,628$ \\
\hline 0.9991 & $€-22,609,005$ & $€-19,661,487$ & $€-47,528,122$ & $€-23,259,649$ \\
\hline 0.9992 & $€-29,447,560$ & $€-34,937,750$ & $€-91,712,158$ & $€-41,462,778$ \\
\hline
\end{tabular}


We conclude that in all cases, given a $99.9^{\text {th }}$ percentile of the risk factor $\alpha_{.999}(X)$, there is a probability $\mathrm{p}>.001$ that banks might be undercapitalized in any given year. The next table shows the decline in the magnitude of the necessary adjustment as $n$ goes up.

Table 5: Magnitude of GA relative to $K+G A$

\begin{tabular}{ccccc}
\hline & $P(1000)$ & $P(2000)$ & $P(3000)$ & $P(6000)$ \\
\hline$G A /(K+G A)$ & $7.8 \%$ & $4.9 \%$ & $3.7 \%$ & $1.6 \%$ \\
\hline
\end{tabular}

Going back to equation (2) we see that the inverse relationship as n grows is confirmed. Thus, while the granularity adjustment is quite significant for the first three portfolios, in terms of $G A /(K+G A)$, as $n$ grows (i.e. $n=6000)$ this ratio tends to become smaller, eventually approaching zero as $n \rightarrow \infty$.

Figure 7 Granularity Adjustment ratio with respect to $K+G A$

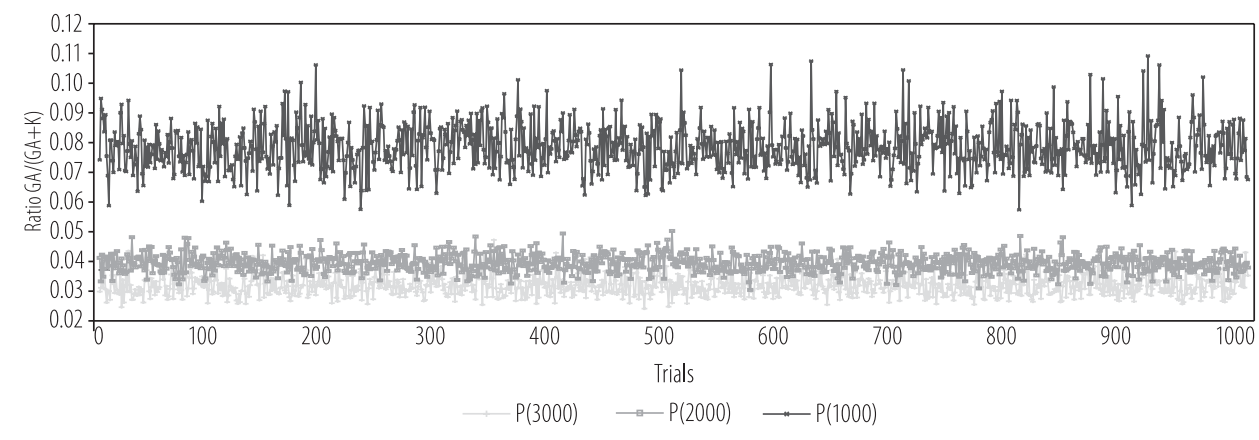

\section{Granularity and Expected Loss}

One additional interesting topic, which has not been addressed by the literature, relates to the levels of expected loss EL for portfolios of differing granularity. The goal of this section is to investigate this effect. Taking the sum over all borrowers we get for $E L$ :

$$
E L=\mathcal{R}=\sum_{i=1}^{n} E U_{i}
$$

As discussed above, the $R$ stands for loan loss provisions and its importance lies in the fact that it represents a significant part of the operational cost of doing business for the FI. It is assumed, corresponding to modern risk-management 
practices, that this amount corresponds to the loss averaged over all possible realizations of the risk factor (corresponding to a typical economic scenario).

As $X \sim N(0,1)$ we set the risk factor's value equal to zero. Differently from the above scenarios, we keep the $P D$ s fixed, i.e. non-stochastic and independent of $X$. Thus, in this simulation we let each borrower default independently with probability $p_{i}=P D_{i}$. The goal is to generate a distribution around the expected loss of the portfolio and analyse the variation around the mean.

\section{Mapping Procedure}

In order to isolate the effect of granularity, after fixing a portfolio, we perform a mapping of both the exposures and the PDs. The PDs (for all i) are set to the exposure weighted average defined as follows:

$$
p^{\prime}=P D_{i}{ }^{\prime}=\sum_{i=1}^{n} \frac{A_{i}}{\sum_{j=1}^{n} A_{j}} P D_{i}
$$

While the exposures are set to the portfolio average:

$$
A_{i}^{\prime}=\frac{\sum_{i=1}^{n} A_{i}}{n}
$$

After performing this mapping, we achieve a uniform portfolio which exhibits no variation in exposure sizes and probabilities of default. The only difference is in the absolute size of the individual exposures, which depends on the number of borrowers in a portfolio.

\section{Results}

Comparing the results from the simulations we see that while EL remains relatively similar between the homogeneous and heterogeneous portfolios (small differences are due to the maturity adjustments), its standard deviation changes significantly. The following table summarizes our findings: 
Table 7: Change in $\sigma_{\mathrm{E}[\mathrm{LGD}]}$ between uniform and heterogeneous portfolios

\begin{tabular}{|c|c|c|c|c|c|c|}
\hline \multirow[t]{2}{*}{ Portfolios } & \multicolumn{2}{|c|}{$P(1000)$} & \multicolumn{2}{|c|}{$P(2000)$} & \multicolumn{2}{|c|}{$P(3000)$} \\
\hline & Heterogeneous & Homogeneous & Heterogeneous & Homogeneous & Heterogeneous & Homogeneous \\
\hline$\mu_{\text {E[LGD] }}$ & $€ 16,802,411,10$ & $€ 17,617,841.40$ & $€ 40,772,237.65$ & $€ 43,006,577.39$ & $€ 59,211,673.62$ & $€ 62,363,175.14$ \\
\hline$\sigma_{E[L G D]}$ & $€ 11,189,068.89$ & $€ 7,187,643.05$ & $€ 20,240,688.90$ & $€ 11,437,979.48$ & $€ 22,123,821.97$ & $€ 12,741,091.32$ \\
\hline$\left(\Delta \sigma_{E[L G D]}\right) / \mu_{E[L G D]}$ & \multicolumn{2}{|c|}{0.23} & \multicolumn{2}{|c|}{0.20} & \multicolumn{2}{|c|}{0.15} \\
\hline
\end{tabular}

Even though variation in the realized losses does not necessarily pose any serious risk to the solvency of the FI (as $K$ would cover such loss), deviations from EL would mean that the FI does not have an optimal amount of provisions and could thus experience liquidity problems in some years, while it would underutilize its assets in others. From the table we see that the variation in the portfolio exposure sizes could cause a deviation in the $E L$ of at least $\geq 15 \%$. Moreover, this does not happen in an adverse, but rather in a normal scenario for the bank. If we assume that given a typical year, the actual loss happens to be one standard deviation away from the mean, this could lead to an additional 65\% higher default costs than expected.

Below are presented graphs corresponding to distributions of homogeneous and heterogeneous portfolios of three different sizes. As can be easily observed, the heterogeneous portfolios display thicker tails than the homogeneous ones, leading to a higher probability for extreme deviations in $E L$.

Figure 8: Expected Loss Distribution for $\mathrm{P}(1000)$

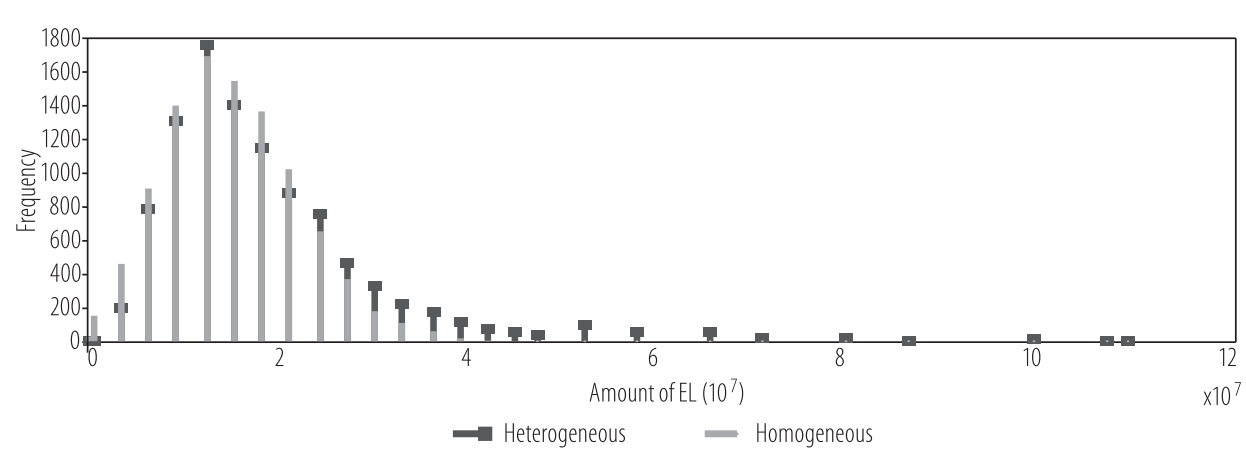


Figure 9: Expected Loss Distribution for $\mathrm{P}(2000)$

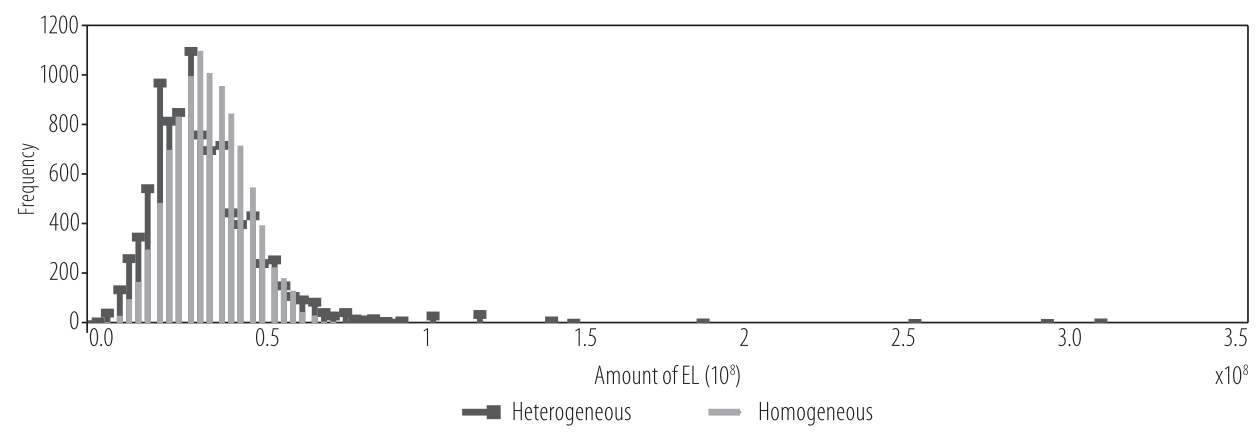

Figure 10: Expected Loss Distribution for $\mathrm{P}(3000)$

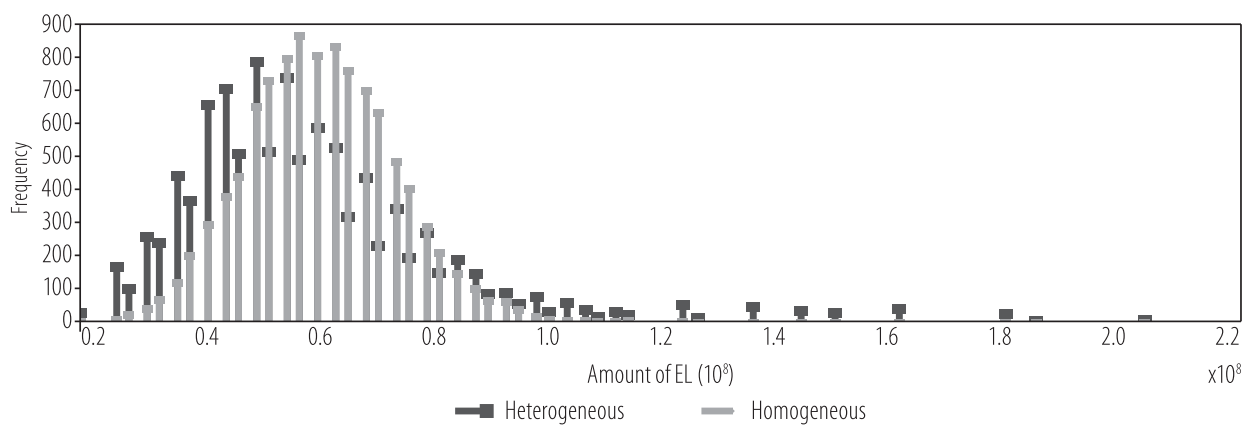




\section{Conclusions}

In this paper we have confirmed the findings of Gordy and Lütkebohmert (2013) about the significance of the granularity adjustment as a discrepancy between the $99.9^{\text {th }}$ percentile of realized losses and the Basel capital requirement. In order to do so we followed two Monte Carlo simulations approaches. In the classic approach we hold the initial portfolio fixed over time. In the second approach, which we consider more realistic, we let the initial portfolio change over time $t$. Both setups confirm that a portfolio's lack of granularity is a significant contributor to the amount of unexpected loss, and thus to capital requirements. We show that, as expected, $G A$ is negatively related to the number of exposures. Furthermore, we find that the variation in expected losses is also significantly affected by the size of the portfolio exposures (non-granularity). Where portfolio nongranularity is present FIs will observe loss amounts that fluctuate considerably around $E L$, leading to inefficient use of resources.

In the presence of non-granular portfolios, a financial institution could opt for adding the granularity adjustment to the actual amount of capital, which would have several consequences. While it could be beneficial for the bank in terms of increased stability (better rating) it could also mean that it is losing ground to its competitors in terms of profitability unless it transfers part (if not all) of the additional capital costs to the obligor. To that end, we argue that it would be appropriate to include a granularity adjustment in the capital requirement component of the loan price. By accounting for the real cost of concentration risk, a first-mover FI could gain a competitive edge in risk-pricing and thus positively influence its performance both in terms of profitability and market share. 


\section{References}

1. Basel Committee on Banking Supervision (2005). An Explanatory Note on the Basel II IRB Risk Weight Functions. Basel: Bank for International Settlements.

2. Basel Committee on Banking Supervision (2006). Studies on Credit Risk Concentration. Basel: Bank for International Settlements.

3. Basel Committee on Banking Supervision (2013). The Liquidity Coverage Ratio and liquidity risk monitoring tools. Basel: Bank for International Settlements.

4. Basel Committee on Banking Supervision (2014). A brief history of the Basel Committee, Basel: Bank for International Settlements.

5. Brůna, K. and Blahová, N. (2016). Systemic Liquidity Shocks and Banking Sector Liquidity Characteristics on the Eve of Liquidity Coverage Ratio Application- The Case of Czech Republic. Journal of Central Banking Theory and Practice, 5(1), 159-184.

6. Credit Suisse Financial Products (1997). CreditRisk+: A Credit Risk Management Framework. London

7. Featherstone, A. M., Roessler, L. M., Barry, P. J. (2006). Determining the Probability of Default and Risk-Rating Class for Loans in the Seventh Farm Credit District Portfolio. Review of Agricultural Economics, 28(1), 4-23.

8. Gordy, M. (2000). A Comparative Anatomy of Credit Risk Models. Journal of Banking \& Finance 24(1-2), 119-149.

9. Gordy, M. (2003). A Risk-Factor Model Foundation for Ratings-Based Bank Capital Rules. Journal of Financial Intermediation 12(3), 199-232.

10. Gordy, M. and Lütkebohmert, E. (2013). Granularity Adjustment for Regulatory Capital Assessment.” International Journal of Central Banking, 9(3), 38-77.

11. Gupton, G. M., Finger, C. C., Bhatia, M. (1997). CreditMetrics-Technical Document. New York: J.P. Morgan \& Co. Incorporated.

12. Merton, R. C. (1974). On The Pricing of Corporate Debt: The Risk Structure of Interest Rates. The Journal of Finance 29(2), 449-470.

13. Stein, R. M. (2005). The Relationship between Default Prediction and Lending Profits: Integrating ROC Analysis and Loan Pricing. Journal of Banking \& Finance 29(5), 1213-1236.

14. Unicredit Bulbank. (2012). Annual Disclosure Year 2012. Retrieved February 05, 2015, from http://www.unicreditbulbank.bg/weblayout/groups/bulbankwebsite/ documents/bbproductdocument/cons_an_disclosure_2012_en_fin.pdf

15. Vasicek, O. (1991). Limiting Loan Loss Probability Distribution. San Francisco: KMV Corporation. 